\title{
Entscheidungen unter
} Unsicherheit in komplexen Systemen

Hermann Held

30.1 Die zentrale Entscheidungsfrage - 306

30.2 Die Tradition des Utilitarismus und die Erwartungsnutzenmaximierung - 307

30.3 Grenzen der Erwartungsnutzen-maximierung angesichts der Klimaproblematik - 308

30.4 Mischformen probabilistischer und nichtprobabilistischer Kriterien - 308

30.5 Das Konzept der starken Nachhaltigkeit: Grenzwerte und die Kosten-Effektivitäts-Analyse - 309

30.6 Konsequenzen für die Interaktion von Politik und Wissenschaft - 310

$30.7 \quad$ Kurz gesagt -311

Literatur - 311 
Wichtige gesellschaftliche Entscheidungen betreffen üblicherweise Handlungen, deren Ziel es ist, an komplexen Systemen Veränderungen vorzunehmen, um das System noch besser auf die Herausforderungen der Zukunft auszurichten. In der Regel lassen sich jedoch die Folgen solcher Entscheidungen nicht genau vorhersagen. Experimentelle Wissenschaften genießen den Vorteil, sich Untersuchungsgegenstände wählen zu können, bei denen immer weiter verfeinerte Experimente die Unsicherheit hinsichtlich der Auswirkungen von Änderungen schließlich „ausreichend“ verringern. Unsicherheit meint hier unvollständiges Wissen, das für die jeweilige Entscheidung relevant ist (Mastrandrea et al. 2010). Entscheider dagegen müssen dem ins Auge sehen, wenn sie bei gegebener Unsicherheit in oft vorgegebener Zeit urteilen und Pläne festlegen sollen. Auch Privatpersonen müssen unter Unsicherheit entscheiden, etwa beim Abschluss von Versicherungen: Es gibt eine Fülle von Angeboten, aber ob ein Angebot genutzt wird und, wenn ja, zu welchen Bedingungen, ist eine persönliche Entscheidung unter Unsicherheit: Soll man mit dem seltenen, aber drohenden möglichen Schaden leben? Oder wäre es besser, die Prämie zu zahlen und so im Mittel Geld zu verlieren - welches das Versicherungsunternehmen im Mittel gewinnt -, um damit einen möglichen finanziellen Großschaden abzuwehren, der die Lebensqualität außergewöhnlich belasten würde? Unsicherheit bei Entscheidungen über die Zukunft zu berücksichtigen wird so zu einem Kernpunkt der Entscheidung selbst (• Abb. 30.1); sie wird „eingepreist“" (Sorger 1999).

Auch ganze Gesellschaften stehen vor Entscheidungen unter Unsicherheit. Ein Beispiel ist, angesichts von Vorhersagen über einen steigenden Meeresspiegel die Deiche zu erhöhen. Die Kosten dafür steigen mit der Höhe; außerdem geht oft ein Verlust an Lebensqualität damit einher, weil die Sicht auf das Meer behindert ist. Wie groß das Überschwemmungsrisiko wirklich wird und wann genau es in Form von Extremereignissen eintritt, ist unklar. Aus Sicht der Küstenländer rührt dies einerseits daher, dass sie die internationale Klimapolitik und damit das Ausmaß des Meeresspiegelanstiegs nicht selbst entscheiden und kaum beeinflussen können. Aber selbst wenn dies der Fall wäre, verblieben erhebliche naturwissenschaftliche und bautechnische Unsicherheiten darüber, welche Investitionen wirklich welchen Rückgang eines Überschwemmungsrisikos bewirken würden. So stellen höhere Deiche eine gewisse Analogie zum Zahlen einer Versicherungsprämie dar. Wie im Folgenden ausgeführt werden wird, können jedoch nicht alle Entscheidungen unter Unsicherheit durch einen Versicherungsansatz gehandhabt werden. Im Folgenden wird daher hervorgehoben, dass es konkurrierende Möglichkeiten, „Entscheidungskriterien“, gibt, Unsicherheit auszudrücken und unter Unsicherheit zu entscheiden. Es wird der Blick dafür geschärft, welche Aspekte bei einer jeweiligen Methode dabei besonders gut oder schlecht im Einklang mit dem Wertesystem der entscheidenden Person stehen könnten.

\subsection{Die zentrale Entscheidungsfrage}

Es gibt formale und daher systematische Möglichkeiten, Unsicherheit darzustellen und unter Einbeziehung dieser Unsicherheiten zu entscheiden. Ein Beispiel stellt die Geschichte der Diskussion des Klimaproblems aus global-wirtschaftlicher Sicht dar. Sie liefert wichtige Hinweise darauf, was die weltweite Klimapolitik antreibt, und zeigt mögliche Potenziale auf, konsistente Handlungen auf regionaler Ebene umzusetzen. Zugleich handelt es sich hierbei um einen besonders stark diskutierten und illustrativen Anwendungsfall für Entscheidung unter Unsicherheit in einem komplexen System. Wie viel Vermeidungsanstrengung ist bei Unsicherheit angesichts eines bestimmten Ziels angemessen? Das ist eine klimapolitisch fundamentale Frage. Eine entscheidungstheoretische Herausforderung des global betrachteten Klimaproblems liegt nun darin, dass wir die Gesamtheit der Klimawandelfolgen derzeit nur sehr schwer abschätzen und bewerten können. Die Vermeidungskosten lassen sich hingegen abschätzen. Dies liegt aus unserer Sicht wesentlich daran, dass das Energiesystem menschengemacht ist. Mit dieser Diskrepanz der Abschätzbarkeiten gilt es im Folgenden umzugehen.

Die Entscheidungstheorie (Sorger 1999) hat verschiedene Verfahren entwickelt, wie bei Unsicherheit so entschieden werden kann, dass der jeweilige Grad des Eingehens auf die unterschiedlichen Zielvorstellungen so gewählt wird, dass die daraus abgeleiteten Handlungsempfehlungen alle Möglichkeiten ausschöpfen und zugleich keine Selbstwidersprüche enthalten. Aus-

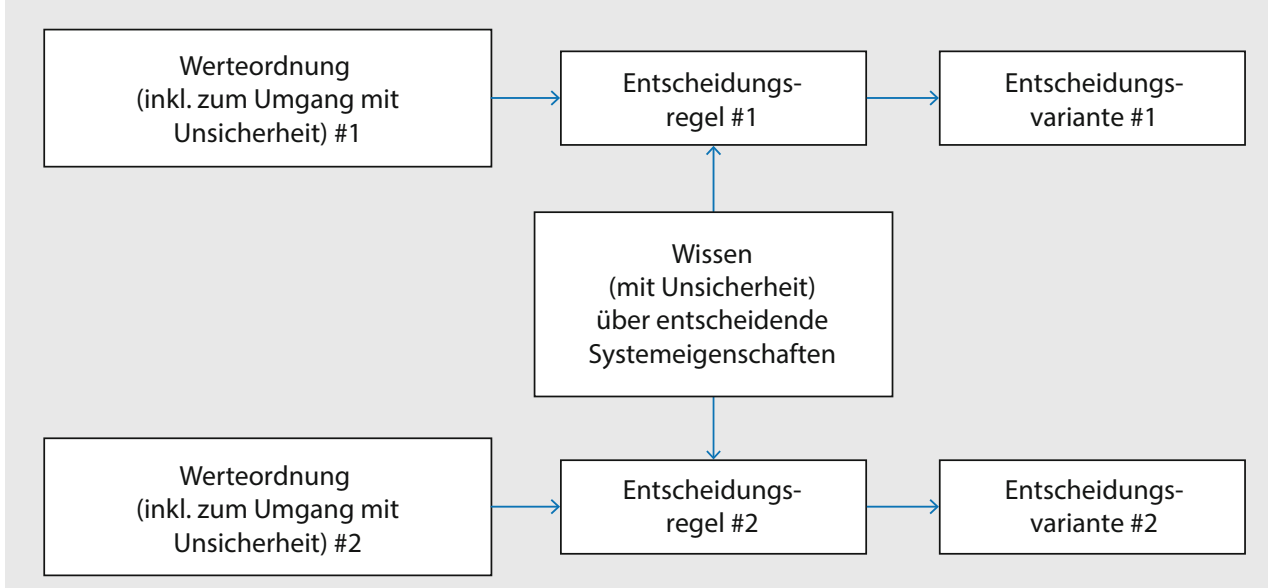

- Abb. 30.1 Wissenschaftliches Wissen über das System kann in Kombination mit gesellschaftlichen normativen Vorgaben zu fundierten Entscheidungen führen. Der Umgang mit Unsicherheit ist hierbei selbst eine normative Vorgabe. Unterschiedliche Normen führen so zu unterschiedlichen Entscheidungen trotz identischer Systemwissensbasis. 
gangspunkt ist immer das Eingeständnis, dass die Folgen unseres Handelns nicht nur von diesem Handeln selbst, sondern auch von bislang noch verborgenen Eigenschaften des Systems abhängen, das wir zu beeinflussen gedenken.

Wie ist nun unter Unsicherheit zu entscheiden? Diese Frage auf gesellschaftlicher Ebene zu beantworten ist selbst schon ein Akt von governance ( Kap. 29). Werden sich die Entscheider zu Unsicherheit etwa eher optimistisch oder pessimistisch verhalten, oder werden sie mit Wahrscheinlichkeiten gewichten? Welche Entscheidungsregel wir wählen, ist eine Vorentscheidung im ethisch-normativen Bereich. Entscheidungstheoretiker haben daher im Dialog mit der Gesellschaft transparent darzulegen, welche Bandbreite an Eigenschaften die jeweiligen Entscheidungsregeln mit sich bringen: Es gibt Hauptannahmen, aber auch überraschende Auswirkungen von Entscheidungen - und ggf. sind neue Regeln vorzuschlagen. Wie bei jeder normativen Vorauswahl fällt der Wissenschaft im Rahmen einer Politikberatung die Aufgabe zu, diejenigen Konsequenzen von Entscheidungen herauszuarbeiten, die sich für die Praxisakteure vermutlich als besonders bedeutsam herausstellen werden. Eben diese Konsequenzen sind dann in der Kommunikation mit Praxisakteuren besonders hervorzuheben. Fühlen sich die stakeholder mit allen Szenarien - d. h. den Konsequenzen möglicher Entscheidungen unwohl, ist der Entscheidungsszenarienpool in einem iterativen Prozess (Edenhofer und Seyboth 2013; s. auch am Ende dieses Kapitels) zu erweitern, um so nach Möglichkeit befriedigendere Lösungen zu suchen. In der Regel wird hier jedoch nur eine Annäherung gelingen, und es werden sich nicht gleichzeitig alle Wünsche befriedigen lassen. Die Wahl des Umgangs mit Unsicherheit ist hierbei Teil dessen, was Entscheider beeinflussen können. Der im - Kap. 29 vorgestellte „Risikodialog am runden Tisch“ kann hierbei ein wirksames Instrument darstellen, sich darüber zu verständigen, wie Unsicherheit bei Entscheidungen Rechnung zu tragen ist.

\subsection{Die Tradition des Utilitarismus und die Erwartungsnutzenmaximierung}

Die derzeit wichtigste Entscheidungsmethode „Erwartungsnutzenmaximierung“ (Sorger 1999) nimmt an, man könne alle verborgenen Systemeigenschaften inklusive aller möglichen Einstellungen benennen und in Zahlen darstellen. Die Gesamtheit dieser möglichen numerischen Einstellungen nennt die Entscheidungstheorie „Weltzustand“. Wüssten wir, wie genau dieser aussieht, könnten wir die Folgen unserer Entscheidungen perfekt voraussagen. Wird dies nun noch in die Tradition des Utilitarismus eingebettet, der auf die Anordnung von möglichen Handlungen entlang einer einzigen numerisch ausdrückbaren Dimension hinausläuft, der sogenannten utility, ergibt sich folgende Weltsicht: Es sind nicht nur alle möglichen Handlungsfolgen vorstellbar, diese können auch mit einem Wahrscheinlichkeitsmaß unterlegt werden. Savage (1954) hat dies aus abstrakten, aber durchaus schlüssigen Theoriegrundsätzen motiviert. Hiernach kann das Wahrscheinlichkeitsmaß subjektiver oder objektiver Natur sein. Wenn es neue objektive Informationen gibt, kann das Maß mithilfe der Bayes-Formel aus dem Bereich der Statistik jeweils auf den neuesten Stand gebracht werden. In dieser Weltsicht wird quasi Unsicherheit als stets durch Wahrscheinlichkeit ausdrückbar verengt. Aus einer Reihe weiterer abstrakter, plausibler Grundsätze folgt, dass sich somit jede Bewertung von Entscheidungen - im Sinne eines Rankings - als Erwartungsnutzenmaximierung ausdrücken lässt.

Es hat sich in der Tradition der Entscheidungstheorie und Ökonomik eingeschliffen, dass derjenige, der stets der Erwartungsnutzenmaximierung folgt, sich entlang des Ideals des „rationalen Entscheiders“ verhalte. Dabei könnte mitschwingen, dass Akteure, die sich entsprechend eines konkurrierenden Entscheidungskriteriums verhalten, irrational, unreflektiert, unlogisch oder intellektuell überfordert sind. Eine unterschwellige Abwertung konkurrierender Entscheidungsmodelle (s. u.) hat somit bereits vor einem offenen Diskurs stattgefunden.

Wie in Abschn. 30.5 ausgeführt wird, kann es jedoch gute Gründe geben, vom Prinzip der Erwartungsnutzenmaximierung abzuweichen (wie dies insbesondere bei der Mehrzahl der $1000 \mathrm{im}$ jüngsten IPCC-Bericht zusammengefassten Szenarien der Fall ist).

Für eine große Klasse von Entscheidungsproblemen macht das Konzept der Erwartungsnutzenmaximierung jedoch Sinn. Dies sei zunächst am Beispiel Deichhöhe und Versicherungen illustriert: Ziel wäre es zu versuchen, die Deichhöhe in einer Gesamtschau von ökonomischer, sicherheitstechnischer und ökologischer Sicht optimal zu bestimmen. Mithilfe von Modellen für den Erfolg internationaler Klimaschutzpolitik müsste eine Wahrscheinlichkeitsverteilung für das weltweite Emissionsverhalten abgeschätzt werden. Aus diesen würde dann mittels downscaling von globalen Klimamodellen eine Wahrscheinlichkeitsverteilung für künftige Sturmfluten ermittelt. Diese würden wiederum mit Überflutungsmodellen in Überflutungskarten übersetzt. Regionalwirtschaftliche Modelle würden daraus schließlich abschätzen, wie sich die geldlich bewerteten Schäden von Überflutungen verteilen. So könnten für verschiedene Deichhöhen der erwartete wahrscheinlichkeitsgemittelte Schaden ermittelt und die Kosten für den notwendigen Deichbau gegengerechnet werden. Es müsste dann die Deichhöhe gewählt werden, welche die Baukosten und die erwarteten vermiedenen Schäden optimiert. Allerdings empfehlen hier Ökonomen noch eine Modifikation: Es sind nicht die monetären Schäden, sondern es ist der "gefühlte Verlust" durch die monetären Schäden in Rechnung zu stellen. Dies gibt die Möglichkeit, seltene, aber große Schäden stärker zu gewichten, wie es auch dem Lebensgefühl der meisten Menschen entspricht. Optimiert wird in der ökonomischen Theorie daher nicht direkt das Monetäre, sondern das durch eine „Nutzenfunktion" gewichtete Monetäre.

Das Konzept der Erwartungsnutzenmaximierung für Entscheidungen bei unsicherer Datenlage ist daher Standard (gerade auch und zu Recht im Versicherungsbereich) und wird meist als zweckmäßig empfunden. Es dominiert die Wirtschaftswissenschaft bis heute. Sollten in einem Entscheidungsfall also tatsächlich die nötigen Eingabegrößen vorliegen, um das Erwartungsnutzenmaximum rechnerisch zu ermitteln, dürfte es kaum Gründe geben, ein anderes Entscheidungskriterium zu wählen. Aber spiegelt dies für den Fall des Klimawandels die Präferenzordnung aller gesellschaftlichen Akteure bestmöglich wider? Das kann bezweifelt werden. Denn das Erwartungsnutzenmaximum 
verlangt, sich alle möglichen Folgen unserer Handlungen vorzustellen und sie mit Wahrscheinlichkeiten zu belegen - ein ehrgeiziges Unterfangen bei komplexen Systemen! Beim Klimaproblem müssten wir uns alle möglichen Folgen des Klimawandels ausmalen, ihre Bewertung mühsam weltweit aushandeln, um die Gewinne und Verluste von Nutzen abschätzen zu können, und noch mit Wahrscheinlichkeiten belegen. Erst dann könnte formal der Erwartungsnutzen (expected utility, EU) maximiert und die „beste" Handlung ausgewählt werden.

\subsection{Grenzen der Erwartungsnutzen- maximierung angesichts der Klimaproblematik}

Dennoch findet diese Erwartungsnutzenmaximierung (EU-Max) in der wirtschaftlichen Betrachtung des Klimaproblems seit etwa 20 Jahren statt. Ein Ergebnis sind z. B. „sozial optimale“ - d.h. wohlfahrtsoptimale - Pfade, die empfehlen, dass die weltweiten Emissionen nur moderat vom bislang üblichen Pfad abweichen mögen. Damit würden sich die Kosten, die entstehen, um den Ausstoß von Treibhausgasen zu verringern, und die Kosten, die entstünden, um die Schäden zu verhindern, die Waage halten. Nordhaus etwa fand noch 2008 optimale Pfade, die eine weltweite Erwärmung von höchstens $3,5^{\circ} \mathrm{C}$ bedeuteten (Nordhaus 2008). Wird die Erwärmung allerdings nur so wenig gebremst, verletzt das die auf den vergangenen Klimakonferenzen vereinbarte Zwei-Grad-Obergrenze eklatant (zu diesem „Zwei-Grad-Ziel“ siehe Schellnhuber 2010).

Ist nun das Zwei-Grad-Ziel „unvernünftig“, weil es den Ergebnissen „rationaler Entscheidungen“ widerspricht, die ein Optimum bei $3,5^{\circ} \mathrm{C}$ angeben? Oder drückt vielmehr das Festhalten am Zwei-Grad-Ziel und dessen Begründung aus, dass sich die Unterstützer dieses Ziels nicht darüber im Klaren sind, was sie dann alles "mitkaufen“ an entscheidungstheoretischen Paradoxien? Aus Sicht der Standard-Entscheidungstheorie wäre das so.

Weitzman (2009) allerdings zeigt aus Sicht des Autors, dass im Zusammenhang mit dem Klimawandel das Erwartungsnutzenmaximum als Entscheidungsgrundlage ungeeignet ist. Modellierte man das Wissen über die Empfindlichkeit des Klimasystems gegenüber Treibhausgaskonzentrationsänderungen konsequenter als bislang im EU-Max-Bezugsrahmen, kombiniert mit einer besonders steil ansteigenden, aber möglichen Schadensfunktion, müssten wir sofort alle Emissionen einstellen: Bei hoher Klimasensitivität würden im (Wahrscheinlichkeits-)Mittel die Folgen derart eklatant sein, dass sie jegliche Kosten der Vermeidung übersteigen würden. Diese enorme Spannbreite an Empfehlungen, die derzeit noch aus dem Kriterium des Erwartungsnutzenmaximums abgeleitet wird, kann nicht als politisch hilfreich bezeichnet werden.

Dass das Erwartungsnutzenmaximum als Kriterium sehr sensibel auf Änderungen von schwer bestimmbaren Eingangsgrößen reagiert - etwa die Wahrscheinlichkeit und das Ausmaß erwarteter Klimaschäden - bemerkten auch Anthoff et al. (2009). Der Standardreflex der Wissenschaftswelt wäre eigentlich gewesen, mehr Forschungsgelder zu fordern, um die aufgezeigten Wissenslücken so schnell wie möglich zu schließen und so das
Standard-Entscheidungsinstrument „EU-Max“ stabil anwenden zu können. Doch dieses käme im Fall des Klimaproblems zu spät: In den kommenden 10 Jahren wird so viel in das weltweite Energiesystem investiert werden, dass dadurch die weltweite Klimaschutzpolitik der kommenden Jahrzehnte im Wesentlichen gebunden sein wird. Es braucht daher ergänzende oder sogar völlig andere Entscheidungskriterien, die mit dem vorhandenen Wissen effizienter zu haushalten verstehen und so schon heute Orientierung für die unmittelbar anstehenden Investitionsentscheidungen bieten können.

Wendet man das Erwartungsnutzenmaximum für komplexe Umweltsysteme an, ist es insbesondere im Umgang mit der Natur sehr schwer, nachvollziehbare Wahrscheinlichkeitsmaße für alle möglichen Weltzustände anzugeben. Der traditionelle Bayesianismus stellt jedoch in den Raum, es sei stets möglich und auch geboten, eine sinnvolle subjektive Wahrscheinlichkeitsverteilung als Standardausgangspunkt für unsicherheitsbehaftete Untersuchungen anzugeben. Gerade wenn es kaum Vorwissen gibt, verwickelt sich dieser Standpunkt jedoch in Widersprüche.

Daher könnte man geneigt sein, Kriterien heranzuziehen, die nicht auf Wahrscheinlichkeitsaussagen fußen. Diese basieren darauf, jeder möglichen Handlung einen besten oder schlimmsten Weltzustand zuzuweisen. In einem zweiten Schritt wird dann entlang der Entscheidungsachse eine bestmögliche Entscheidung vorgeschlagen (Sorger 1999): Ein Optimist würde z. B. von einer Klimasensitivität nahe Null ausgehen und bräuchte sich folglich nicht um das Klimaproblem zu kümmern. Das minimum-regretKriterium, das Kriterium „des geringsten Bedauerns“, schließlich minimiert den maximal möglichen Nutzenabstand gegenüber einem imaginierten Akteur mit perfekter Information. Sogenannte „robuste “ Kriterien nutzen dann einen Verschnitt aus EU-MaxAspekten für Systemkomponenten, die besser verstanden sind, und aus Kriterien, die nicht auf Wahrscheinlichkeitsaussagen basieren, für weniger gut verstandene Komponenten (Lempert et al. 2006; Hall et al. 2012).

\subsection{Mischformen probabilistischer und nichtprobabilistischer Kriterien}

Konzepte, die nicht allein auf Wahrscheinlichkeiten fußen, konzentrieren sich auf die Extreme: Was kann im besten und was im schlechtesten Fall passieren? Daher weisen sie beim Klimaproblem die Schwierigkeit auf, dass sie letztlich in radikale Empfehlungen („Nichtstun“ oder „Einstellen jeglicher Emission“) münden würden, denn mit je einer gewissen Wahrscheinlichkeit könnten Folgen von Treibhausgasemissionen auch vernachlässigbar oder aber quasi unbegrenzt sein. Solche radikalen Empfehlungen dürften allerdings kaum die gesellschaftliche Präferenzordnung widerspiegeln. Vielleicht sind beide Ansätze, eine Wahrscheinlichkeitsverteilung unter allen Umständen wie beim Erwartungsnutzenmaximum und das völlige Absehen davon, also überhaupt eine Gewichtung unbestimmter Messgrößen anzugeben, zu radikal und unangemessen? Womöglich liegt das angemessene Modell, unser Wissen auszudrücken, in einem stetigen Übergang zwischen beiden? Derartige Modelle sind entwickelt worden. In der prominentesten Version lässt sich unser Wissen nicht mithilfe 
jeweils einer einzigen Wahrscheinlichkeitsverteilung ausdrücken, sondern eher mit einem Bündel von Verteilungen (Walley 1991).

So vielversprechend dieser Zugang ist, unsicheres Vorwissen zu modellieren, so wirft er doch neue Paradoxa auf (z. B. Walley 1991; Held und Edenhofer 2008). Der Autor tendiert dazu zu empfehlen, denselben zunächst in seinen Konsequenzen weiter zu untersuchen, bevor er in der Politikberatung eingesetzt würde.

\subsection{Das Konzept der starken Nachhaltigkeit: Grenzwerte und die Kosten-Effektivitäts- Analyse}

Dies lenkt den Blick auf eine radikal einfachere Lösung: das alte, umweltpolitisch etablierte Konzept von Grenzwerten, die nicht überschritten werden sollten, auch bekannt als Konzept der „starken Nachhaltigkeit“ (Hediger 1999). Wo genau die Grenzwerte jeweils liegen sollen, hängt vom Wissen und von den Normen der beteiligten Akteure ab. Anlass zur Wahl von Grenzwerten sind Situationen der folgenden Kategorien:

1. Das Überschreiten einer naturgegebenen Schwankungsbreite: Mangels Systemwissen wird angenommen, dass sich eine Auswirkung „in Grenzen“ halten könnte, wenn der Eingriff sich im Rahmen der natürlichen Schwankungsbreite bewegt;

2. Tipping points (Lenton et al. 2008): objektiv gegebene Schwellenwerte, deren Überschreitung langfristig einen „völlig anderen" Systemzustand zur Folge hätte, oder

3. wie beim Zwei-Grad-Ziel um einen politisch gesetzten, jedoch mit akademischem Wissen unterlegten Grenzwert (analog zur einer Geschwindigkeitsbegrenzung im Verkehrsbereich). Das Zwei-Grad-Ziel stellt nach Auffassung des Autors ein Hybrid aus den folgenden drei Begründungssträngen dar: - Es gibt bereits bekannte und ökonomisch bewertete Klimawandelfolgen, die die Gesellschaft aus Sicht der Proponenten des Zwei-Grad-Ziels vermeiden sollte.

- Zugleich wird davon ausgegangen, dass wir bei Weitem nicht alle Folgen der Erwärmung kennen. Hier kann gefragt werden, wie stark die globale Mitteltemperatur im Rahmen der Erdgeschichte natürlicherweise schwanke und ob menschengemachte Erwärmung groß oder klein im Vergleich dazu sei. Aus einer Vorsorgeperspektive heraus könnte man dann fordern, den menschengemachten Beitrag in diesem Sinne „klein“ zu halten.

- Schließlich benötigt der politische Aushandlungsprozess stark kondensierte Zielvorstellungen; hier kann die Angabe einer möglichst einprägsamen, glatten, wenngleich akademisch informierten Zahl hilfreich sein. (Nicht gemeint ist hingegen ein tipping point für das gesamte Klimasystem, bei dem nach einer noch so kleinen Überschreitung die globale „Katastrophe“ sicher unausweichlich wäre.)

Ist ein Grenzwert festgesetzt, wird das Erwartungsnutzenmaximum durch ein bedingtes Erwartungsnutzenmaximum ersetzt, das diesen Grenzwert einhält: die Kosten-Effektivitäts-Analyse (KEA). Man fragt, welche Politik es erlauben würde, den Grenzwert mit dem geringsten ökonomischen Aufwand einzuhalten.
Entscheidend ist hierbei, den Teil der Analyse, der die Wissenschaftswelt bis auf Weiteres überfordert, für eine Entscheidungsfindung nicht zu benötigen. Setzt man etwa ein Zwei-Grad-Ziel, braucht man im Anschluss die Klimawandelfolgen nicht $\mathrm{zu}$ modellieren, sondern „nur“ die Transformation des (besser verstandenen) Energiesystems, das zu einer Zwei-Grad-Welt führen kann.

Dieses Vorgehen ist dann sinnvoll, wenn

I. man der Meinung ist, Klimawandelfolgen noch nicht annähernd vollständig abschätzen zu können, die Kosten des präventiven Zwei-Grad-Ziels jedoch schon;

II. die Kosten zur Erreichung des Zwei-Grad-Ziels als „klein“ angesehen würden. Aus Sicht von immer mehr Wirtschaftsexperten treffen diese beiden Voraussetzungen zu:

- (I) ist erfüllt, weil es einfacher ist, das Energiesystem zu modellieren als die natürliche Umwelt, denn das Energiesystem ist weniger komplex, menschengemacht und menschengesteuert.

- Zu (II) berichtet der IPCC zusammenfassend, das ZweiGrad-Ziel bedeute, das globale Wirtschaftswachstum um 0,06 Prozentpunkte pro Jahr zu senken - gegenüber einer Erwartung von 1,6-3\% pro Jahr (Edenhofer et al. 2014). Viele Akteure dürften diese Zahl als „klein“ einstufen.

So könnte eine Gesellschaft über Grenzwerte und eine KostenEffektivitäts-Analyse zu Handlungen kommen, selbst wenn das gekoppelte Gesamtsystem noch nicht bewertet werden kann (Patt 1999; Held und Edenhofer 2008).

Starke Nachhaltigkeit und damit harte Grenzen als handlungsleitende Prinzipien münden jedoch in konzeptionelle Schwierigkeiten, sollte man mit der Möglichkeit rechnen müssen, dass der Grenzwert irgendwann nicht mehr einzuhalten wäre. Dieses kann auftreten, weil Handlungen verzögert wurden oder weil das System viel empfindlicher auf Eingriffe reagiert als erwartet.

Für den vorliegenden Fall, in dem die Situation eher der Kategorie „3“ oder „1“ denn „2“ (s. o.) zuzurechnen ist, haben Schmidt et al. (2011) vorgeschlagen, das „Risiko“ der Grenzüberschreitung mit den Aufwendungen für Klimaschutz zu verrechnen (Schmidt et al. 2011). Diese „weichere“ Variante eines umweltpolitischen Ziels in Kombination mit KEA nennen sie „Kosten-Risiko-Analyse“. Neubersch et al. (2014) weisen aus, dass sich im Fall des Zwei-Grad-Ziels dann etwa dieselben Handlungsempfehlungen ergäben wie infolge der noch standardmäßig verwendeten KEA. Bei anderen Anwendungen mag es jedoch größere Differenzen geben.

Anwender sollten darauf achten, ob künftiges Lernen über Systemantworten für ihren Entscheidungshorizont relevant ist. Wenn dies der Fall sein sollte, könnte es sinnvoll sein, von Anfang an mit der Kosten-Risiko-Analyse zu arbeiten. Der Autor erwartet, dass sie eine wichtige und einfach zu implementierende „Brückentechnologie“ sein könnte, solange Formalismen, die kontinuierlich zwischen probabilistischem Wissen und Nichtwissen vermitteln würden ( Abschn. 30.4), nicht voll ausgearbeitet und verstanden sind. Das neue Instrument hat einen weiteren Vorteil gegenüber der älteren, strikten Interpretation: Die 


\begin{tabular}{|c|c|c|c|}
\hline Regel & Art des Ansatzes & $\begin{array}{l}\text { Umgang mit } \\
\text { Unsicherheit }\end{array}$ & Ziel \\
\hline EU-Max & probabilisitsch & $\begin{array}{l}\text { Subjektive } \\
\text { Wahrscheinlichkeitsannahmen } \\
\text { mit objektivierbarem } \\
\text { Hinzulernen }\end{array}$ & $\begin{array}{l}\text { Maximale erwartete (im } \\
\text { Sinne von: wahrscheinlich- } \\
\text { keitsgewichtete) Nutzen- } \\
\text { funktion }\end{array}$ \\
\hline Optimismus/Pessimismus & nichtprobabilistisch & $\begin{array}{l}\text { Vorstellen \& Selektion des } \\
\text { best/schlechtestmöglichen } \\
\text { Weltzustandes (keine } \\
\text { Wahrscheinlichkeitsangaben) } \\
\text { pro Handlung }\end{array}$ & $\begin{array}{l}\text { Maximale Nutzenfunktion, } \\
\text { nachdem für jede mögliche } \\
\text { Handlung optimistisch/ } \\
\text { pessimistisch der } \\
\text { Weltzustand selektiert } \\
\text { wurde }\end{array}$ \\
\hline Minimum regret & nichtprobabilistisch & $\begin{array}{l}\text { Vorstellen \& Selektion des } \\
\text { Weltzustandes pro Handlung, } \\
\text { bei dem das Bedauern am } \\
\text { geringsten ausfiele, nachdem } \\
\text { der wahre Zustand dann doch } \\
\text { gelernt worden wäre }\end{array}$ & $\begin{array}{l}\text { Maximale Nutzenfunktion } \\
\text { nach Zustandsselektion }\end{array}$ \\
\hline $\begin{array}{l}\text { Kosten-Effektivitäts-Analyse } \\
\text { (KEA) }\end{array}$ & $\begin{array}{l}\text { Mischform; benötigt } \\
\text { Grenzwert (z.B. Zwei-Grad- } \\
\text { Ziel) }\end{array}$ & $\begin{array}{l}\text { Ausgrenzen des Bereiches } \\
\text { größter Unsicherheiten - diese } \\
\text { werden als jenseits des } \\
\text { Grenzwerts liegend } \\
\text { angenommen }\end{array}$ & $\begin{array}{l}\text { Maximale erwartete } \\
\text { Nutzenfunktion vor dem } \\
\text { Grenzwertes }\end{array}$ \\
\hline Kosten-Risiko-Analyse & probabilistisch & $\begin{array}{l}\text { Wie KEA, jedoch Einpreisung } \\
\text { der Überschreitung des } \\
\text { Grenzwerts; Eichung an bereits } \\
\text { politisch gesetzten Klimazielen }\end{array}$ & Wie EU-Max \\
\hline
\end{tabular}

- Abb. 30.2 Übersicht zu Entscheidungsregeln und ihren Merkmalen, sowohl auf Wahrscheinlichkeitsannahmen basierend (probabilistisch) als auch unabhängig davon. (Eigene Darstellung des Autors)

darin verwendete „weichere“ Interpretation des Zwei-Grad-Ziels erlaubt es, dieses Ziel notfalls moderat zu überschreiten (falls es wegen weiterhin nicht umgesetzten globalen Klimaschutzes nicht anders möglich ist), das zugrunde liegende Normensystem jedoch aufrechtzuerhalten: Die Zwei-Grad-Community bliebe handlungsfähig, auch wenn das Ziel nicht mehr exakt einzuhalten wäre (• Abb. 30.2).

\subsection{Konsequenzen für die Interaktion von Politik und Wissenschaft}

Was bedeutet die vorliegende Analyse nun für Verwaltung und Politik? Der Umgang mit Unsicherheit bedarf auch normativer Setzungen. Die Verantwortung dafür, welche Normen governance bestimmen sollten, tragen Politik und Verwaltung. Diese Entscheidung kann gerade nicht von den Wissenschaftlern übernommen werden. Aber die Wissenschaft kann der Politik und Verwaltung ein reicheres und transparenteres Spektrum an ausgearbeiteten Vorschlägen für Normen und entsprechende Szenarien zur Verfügung stellen und auf den normativen Entscheidungsbedarf, der sich spezifisch aus Unsicherheit ergibt, hinweisen. In diesem Zusammenhang sei noch einmal auf das jüngste Politikberatungsmodell der Arbeitsgruppe III im Fünften Sachstandbericht des Weltklimarats (IPCC-WG3) verwiesen, das sogenannte „erleuchtet pragmatische Modell“ (Edenhofer und Seyboth 2013; Edenhofer und Kowarsch 2015). Dementsprechend können weder Wissenschaftler noch die Gesellschaft unabhängig voneinander langfristige Ziele festlegen. Vielmehr würde die Gesellschaft normative Vorstellungen (Umweltziele, Umgang mit Unsicherheit) formulieren und die Wissenschaft deren Konsequenzen anhand von Szenarien illustrieren. In deren Lichte könnte die Gesellschaft ihre normativen Forderungen überdenken und ggf. revidieren, weil sie sich weiterer Zielkonflikte bewusst geworden wäre. Dieser Zyklus würde idealerweise bis zur vollständigen Konvergenz durchlaufen. Zunächst überzeugende Dringlichkeiten und weniger Wichtiges werden so nach und nach immer grundsätzlicher und fundamentaler geordnet. Mit Unsicherheit umzugehen ist dann nur ein Spezialfall unter vielen anderen normativen Aspekten, zu denen sich die Gesellschaft zu äußern hat. (Für die Wissenschaft ist hierbei wichtig, sich für das gesamte normative Spektrum zu öffnen, statt sich jeweils in die Denkweise einer einzigen Schule einzukapseln.)

Das Konzept zur Risikosteuerung des Internationalen Risikorats (International Risk Governance Council, IRGC) ( Kap. 29) 
wäre demnach mehrfach zu durchlaufen - ein überaus ehrgeiziges Unterfangen!

\subsection{Kurz gesagt}

Bei den meisten Entscheidungen, die komplexe Umweltsysteme betreffen, spielt Unsicherheit eine entscheidende Rolle. Dies ist besonders aus Sicht regionaler Akteure dann der Fall, wenn es abzuwägen gilt, wie genau man sich gegen schwer abzuschätzende Folgen des Klimawandels schützen soll. Das wirtschaftliche Standardinstrument der Erwartungsnutzenmaximierung kann versagen, solange das Wissen über das System mit teils großen Unsicherheiten behaftet ist. Das zeigen die Abwägungen des Klimaziels selbst. Dann ist zu prüfen, ob nicht z. B. kombinierte Entscheidungskriterien wie eine flexibilisierte KostenEffektivitäts-Analyse („Kosten-Risiko-Analyse“) die Präferenzen der Entscheider besser repräsentiert. Die Wahl, nach welcher Methode unter Unsicherheit entschieden werden soll, ist bereits eine normative Vorentscheidung. Wie bei allen Entscheidungen über komplexe Systeme könnten Entscheider sie sinnvoll fällen, nachdem ein iterativer und transparenter Dialogprozess zwischen Entscheidern, Akteuren verschiedener gesellschaftlicher Gruppen und der Wissenschaft aktiv betrieben wurde.

\section{Literatur}

Anthoff D, Tol RSJ, Yohe GW (2009) Risk aversion, time preference, and the social cost of carbon. Environ Res Lett 4:024002

Edenhofer O, Kowarsch M (2015) Cartography of policy paths: a model for solution-oriented environmental assessments. Environ Sci Pol 51:56-64

Edenhofer O, Seyboth K (2013) Intergovernmental panel on climate change. Shogren JF (Hrsg) Encyclopedia of energy, Natural resource and environmental economics 1: ENERGY, 48-56

Edenhofer O, Pichs-Madruga R, Sokona Y, Farahani E, Kadner S, Seyboth K, Adler A, Baum I, Brunner S, Eickemeier P, Kriemann B, Savolainen J, Schlomer S, von Stechow C, Zwickel T, Minx JC (2014) Climate Change. Mitigation of climate change, Summary for Policymakers, Contribution of Working Group III to the Fifth Assessment Report of the Intergovernmental Panel on Climate Change. Cambridge University Press, Cambridge

Hall JW, Lempert RJ, Keller K, Hackbarth A, Mijere C, Mclnerney DJ (2012) Robust climate policies under uncertainty: a comparison of robust decision making and info-gap methods. Risk analysis. http://onlinelibrary.wiley.com/ doi/10.1111/j.1539-7 6924.2012.01802.x/full

Hediger W (1999) Reconciling "weak" and "strong" sustainability. Int J Soc Econ 26:1120-1143

Held $\mathrm{H}$, Edenhofer $\mathrm{O}$ (2008) Re-structuring the problem of global warming mitigation: „climate protection“ vs. „economic growth" as a false trade-off. In: Hirsch Hadorn G, Hoffmann-Riem H, Biber-Klemm S, GrossenbacherMansuy W, Joye D, Pohl C, Wiesmann U, Zemp E (Hrsg) Handbook of transdisciplinary research. Springer, Heidelberg, S 191-204

Lempert RJ, Groves DG, Popper SW, Bankes SC (2006) A general, analytic method for generating robust strategies and narrative scenarios. Manage Sci 52:514-528

Lenton TM, Held H, Kriegler E, Hall J, Lucht W, Rahmstorf S, Schellnhuber HJ (2008) Tipping elements in the Earth's climate system. PNAS 105(6):17861793

Mastrandrea M, Field C, Stocker T, Edenhofer O, Ebi K, Frame D, Held H, Kriegler E, Mach K, Matschoss P et al (2010) Guidance note for lead authors of the IPCC fifth assessment report on consistent treatment of uncertainties. Intergovernmental Panel on Climate Change, Genf
Neubersch D, Held H, Otto A (2014) Operationalizing climate targets under learning: an application of cost-risk analysis. Clim Chang 126(3):305-318. doi:10.1007/s10584-014-1223-z

Nordhaus WD (2008) A question of balance: economic modeling of global warming. Yale University, New Haven

Patt A (1999) Separating analysis from politics. Policy Stud Rev 16(3-4):104-137

Savage LJ (1954) The foundations of statistics. Wiley, New York

Schellnhuber HJ (2010) Tragic triumph. Clim Chang 100:229-238. doi:10.1007/ s10584-010-9838-1

Schmidt MGW, Lorenz A, Held H, Kriegler E (2011) Climate targets under uncertainty: 34 challenges and remedies. Clim Chang 104:783-791

Sorger G (1999) Entscheidungstheorie bei Unsicherheit. Lucius \& Lucius, Stuttgart

Walley P (1991) Statistical reasoning with imprecise probabilities. Chapman \& Hall, London

Weitzman ML (2009) On modeling and interpreting the economics of catastrophic climate change. Rev Econ Stat 91:1-19

Open Access Dieses Kapitel wird unter der Creative Commons Namensnennung 4.0 International Lizenz (http://creativecommons.org/ licenses/by/4.0/deed.de) veröffentlicht, welche die Nutzung, Vervielfältigung, Bearbeitung, Verbreitung und Wiedergabe in jeglichem Medium und Format erlaubt, sofern Sie den/die ursprünglichen Autor(en) und die Quelle ordnungsgemäß nennen, einen Link zur Creative Commons Lizenz beifügen und angeben, ob Änderungen vorgenommen wurden.

Etwaige Abbildungen oder sonstiges Drittmaterial unterliegen ebenfalls der genannten Creative Commons Lizenz, sofern sich aus der Abbildungslegende oder der Quellreferenz nichts anderes ergibt. Sofern solches Drittmaterial nicht unter der genannten Creative Commons Lizenz steht, ist eine Vervielfältigung, Bearbeitung oder öffentliche Wiedergabe nur mit vorheriger Zustimmung des betreffenden Rechteinhabers oder auf der Grundlage einschlägiger gesetzlicher Erlaubnisvorschriften zulässig. 